\title{
Sjogren's syndrome associated with bilateral peripheral facial paralysis
}

\author{
Kubra Isik, MD, Gulin Morkavuk, MD, Guray Koc, MD, Zeki Odabasi, MD.
}

\begin{abstract}
متلازمة سجوجرن (SS) هو اضطراب مناعي ذاتي مزمن قد الد يكون معقدًا

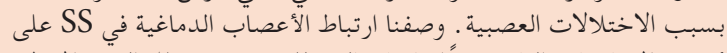

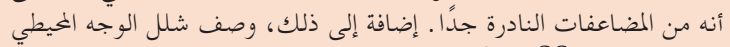

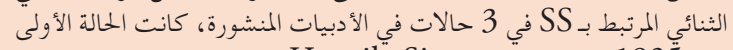

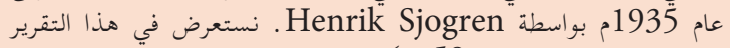

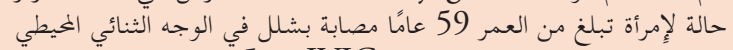

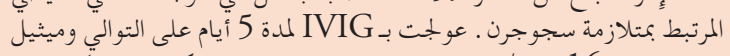

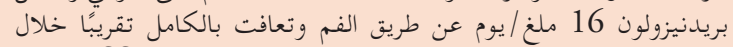

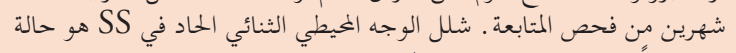

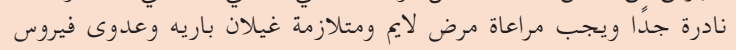

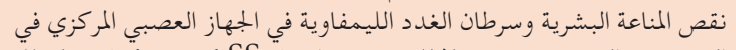

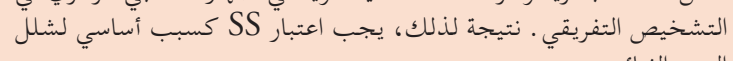

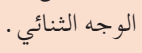

Sjogren's Syndrome (SS) is a chronic autoimmune disorder that may be complicated by neurological dysfunctions. The involvement of cranial nerves in SS was described as a very rare complication. Moreover, bilateral peripheral facial paralysis associated with SS has been described only in 3 patients in the literature and the first case was described by Henrik Sjogren himself in 1935 . We report a 59-year-old female with bilateral peripheral facial paralysis associated with Sjogren's syndrome. She was treated with 5-day IVIG consecutively and continued oral methylprednisolone $16 \mathrm{mg} /$ day and almost fully recovered at 2 months of follow-up examination. Acute bilateral peripheral facial palsy in SS is a very rare condition and Lyme disease, Guillain-Barré syndrome, HIV infection, and central nervous system lymphoma should be considered in the differential diagnosis. As a result, SS should be considered as an underlying cause of bilateral facial paralysis.

Neurosciences 2021; Vol. 26 (4):389-391 doi: 10.17712/nsj.2021.4.20210036

From the Department of Neurology (Isik, Morkavuk), Faculty of Medicine, Ufuk University, and from the Department of Neurology (Odabasi), University of Health Sciences, Gulhane Medical School Ankara, and from Department of Neurology (Koc), Istanbul Doctor Lutfi Kirdar Kartal Training and Research Hospital, Istanbul, Turkey

Received 4th April 2021. Accepted 29th July 2021.
Address correspondence and reprint request to: Dr. Gulin Morkavuk, Department of Neurology, Ufuk University, Faculty of Medicine, Ankara,Turkey.E-mail:drgcmor@yahoo.com

ORICID ID: https://orcid.org/0000-0001-7522-8585

Sögren's Syndrome (SS) is a systemic chronic $\checkmark$ autoimmune and vasculitic disorder characterized by lymphocytic infiltration of lacrimal and salivary glands. ${ }^{1}$ In $39 \%$ of the affected cases, the disease can start with neurological findings. The most common peripheral involvement of SS is sensory neuropathies. ${ }^{2}$

Peripheral facial paralysis associated with primary SS has been described in very few cases, and most of them were unilaterally affected. ${ }^{3}$ Only three had bilateral facial nerve involvement. ${ }^{4}$ Here, we described a patient with SS, who presented with bilateral facial nerve involvement.

Case Report. Patient information and clinical findings. A 59-year-old female patient was admitted to our clinic with complaints of inability to close her eyes and raise her eyebrows. Neurological examination showed bilateral difficulty in raising eyebrows and inability to close the eyes. But the mouth was spared. In the motor and sensorial examination, there was no lateralized deficit, and deep tendon reflexes were normoactive. The patient was hospitalized at our clinic.

The patient who came to our clinic had been diagnosed with rheumatoid arthritis 10 years earlier. Four years after diagnosis of rheumatoid arthritis, because she was positive for SS-A and SS-B antibodies, she was followed up with a diagnosis of undifferentiated connective tissue disease and was prescribed methotrexate and steroids that she used for 6 years. After her SS-A and SS-B antibodies became negative,

Disclosure. The authors declare no conflicting interests, support or funding from any drug company. 


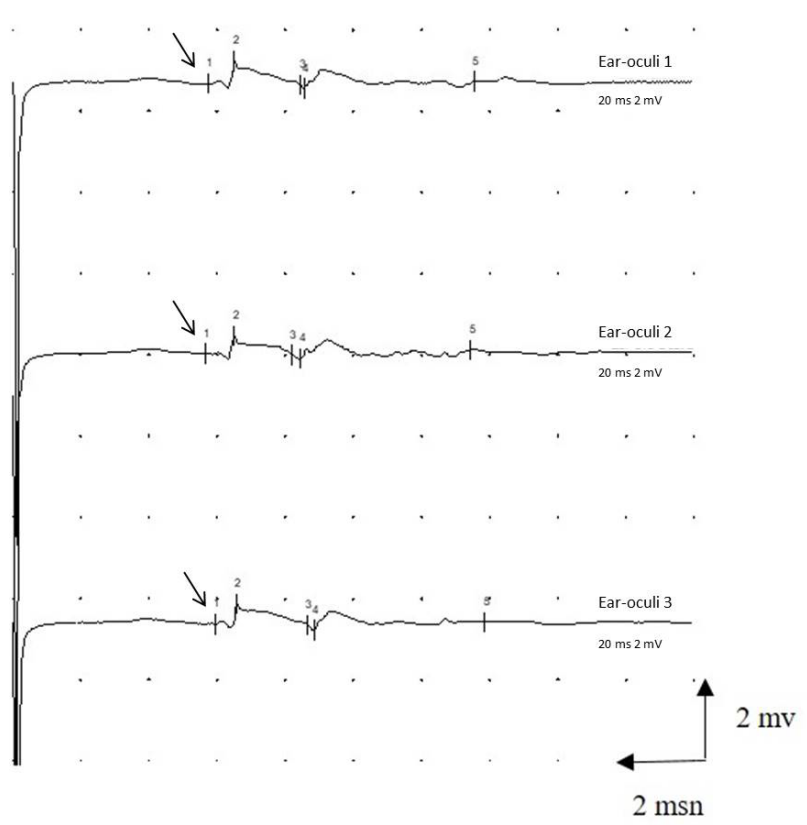

Figure 1 - Prolonged distal motor latency (arrow) and decreased amplitude of the compound muscle action potential (CMAP) recorded in the orbicularis oculi muscle by needle. The CMAP was recorded three times for confirmation

only low-dose steroids were given whereas methotrexate was cut.

Diagnostic assessment and therapeutic intervention. Facial nerve electroneuromyography (EMG) was performed on the 6th day after beginning of the symptoms. Nerve conduction study (NCS) of facial nerve showed prolonged distal motor latency (5.9 $\mathrm{ms}, \mathrm{N}<3 \mathrm{~ms}$ ) and, dispersion and decreased amplitude $(0.7 \mathrm{mV}, \mathrm{N}>3 \mathrm{mV})$ of the compound muscle action potential (CMAP) in the orbicularis oculi and frontal muscles but orbicularis oris (Figure 1). Therefore, NCS of the facial nerve revealed subtotal axonal degeneration in the branches of the bilateral facial nerve which innervated frontalis and orbicularis oculi muscles. Blink reflex latencies were also prolonged. NCS was normal in the right median, ulnar, sural, peroneal and tibial nerves and no signs of peripheral neuropathy were detected.

No signs of peripheral neuropathy were detected. Brain MRI revealed a $2-\mathrm{cm}$ transverse-diameter focal lesion that was hyperintense signaling in T2A and FLAIR sequences in the splenium section of the corpus callosum, increased linear contrast-enhancing in the trace of the bilateral facial nerve and the geniculate ganglion (Figure $2 \mathrm{~A}-\mathrm{B}$ ).

A lumbar puncture was performed on the patient to investigate the etiology of bilateral facial paralysis. No
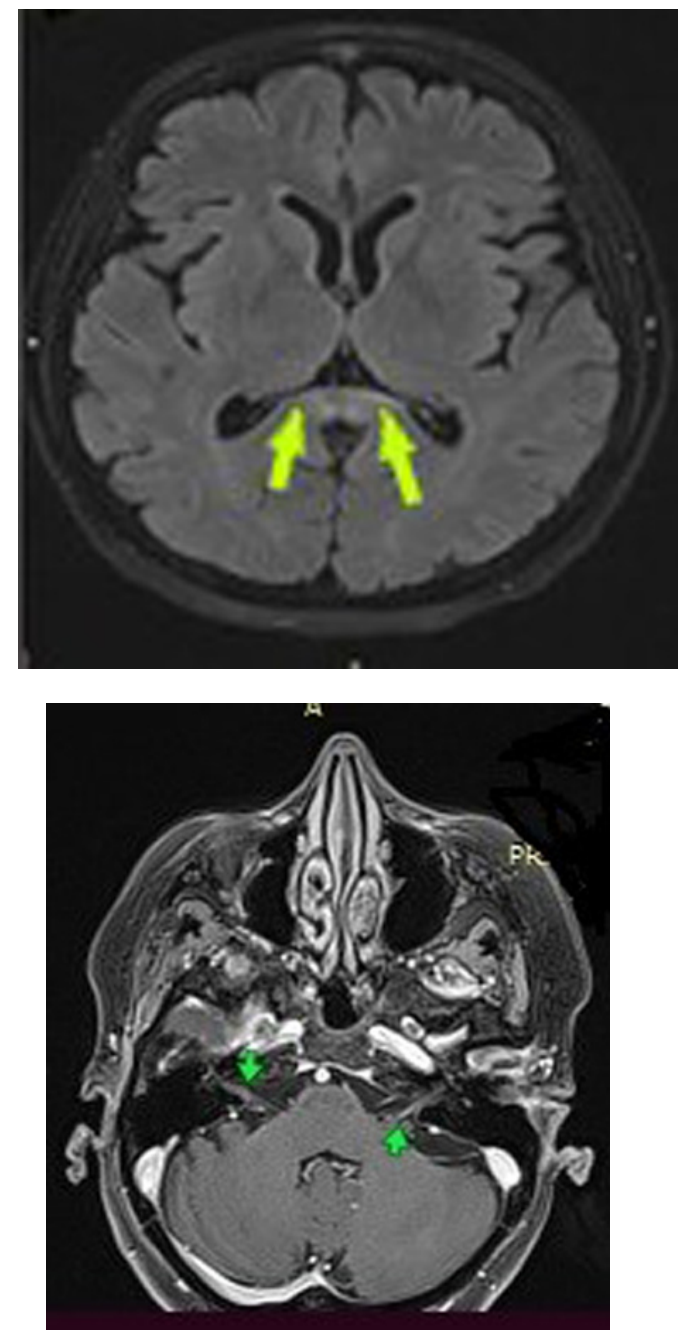

Figure 2 - Cranial MRI image of the patient A) Splenium involvement, B) Arrows show facial nerve enhancement bilaterally.

cells were seen on direct microscopic examination of the cerebrospinal fluid (CSF). Protein $(36,3 \mathrm{mg} / \mathrm{dL})$ and glucose $(59 \mathrm{mg} / \mathrm{dL})$ in the CSF were normal. Borrelia and brucella antibodies were negative in the CSF. The CSF angiotensin converting enzyme (ACE) $(<9$ U/L) and serum ACE $(23 \mathrm{mg} / \mathrm{dL})$ were normal. Sarcoidosis was ruled out by normal serum and CSF ACE values and absence of a contrast-enhancing parenchymal or leptomeningeal lesion on cranial MRI. When the patient applied to our clinic, she also had a dry mouth and dry eyes. Therefore, anti-DS DNA, anti-RNP, and anti-SM were found to be positive. When all the tests performed were reviewed, it was thought that it might be SS. Schirmer test was performed with rheumatology recommendation. Schirmer's test measure was $4 \mathrm{~mm}$ wetting per $5 \mathrm{~min}$. 30g/day i.v. immunoglobulin (IVIG) 
treatment was started for the patient. The treatment continued for 5 days. The patient was discharged with an oral steroid.

Follow-up and outcomes. Two months later, when she came for a follow-up examination, she had made an almost full recovery. The patient gave informed consent for publication of this case report.

Discussion. Sjogren's Syndrome is an autoimmune disorder. It is characterized by keratoconjunctivitis sicca, xerostomia, other connective tissue diseases triad. ${ }^{3}$ It often progresses with peripheral nervous system involvement. Colaci et $\mathrm{al}^{3}$ reviewed the literature on cranial neuritis in SS. When cranial nerve involvement occurred in patients with SS, optic neuritis was the most frequent at $46 \%$ followed by trigeminal neuropathy at $38 \%$. In this study, facial paralysis has been described in 23 cases. Ten among these were multiple cranial neuropathies, 13 were pure facial paralysis, and only 2 were bilateral facial paralysis. The first was defined in 1935 by Sjogren. ${ }^{3}$ Finally, 2 bilateral facial paralysis cases due to Sjogren Syndrome were reported in Germany in 2019 and 2020.4,5 Our case is the 4th. According to these studies, none of the patients had bilateral forehead facial paralysis. Interestingly enough, our patient's facial paralysis included the forehead, sparing the mouth. It is known that trigeminal neuropathy is due to ganglionopathy. However, the pathophysiological mechanisms underlying other cranial neuropathies have not been clearly elucidated. ${ }^{6}$ While periventricular and subcortical white matter lesions are seen more frequently in cranial imaging in SS, corpus callosum lesions can be seen rarely. Brain MRI of the patient revealed geniculate ganglion involvement which cannot explain the clinic of the patient. It is unclear if the corpus callosum lesion is due to SS.

Bilateral facial paralysis is rare. It may be a symptom of Lyme disease, Guillain-Barré syndrome, sarcoidosis, HIV infection, and central nervous system lymphoma. Therefore, above mentioned diseases, were ruled out. ${ }^{7}$ Gaudin et $\mathrm{al}^{7}$ investigated 68 patients with bilateral facial paralysis, and it was mostly seen in Lyme disease as well as in Möbius syndrome. Also, SS must be considered as a cause of bilateral facial paralysis as it was in our case.
A comprehensive physical examination together with appropriate imaging studies and blood work should be performed to establish the correct diagnosis. ${ }^{8}$

Immunosuppressive and immunomodulatory treatments are used for neurological involvement of Sjogren syndrome. Steroid and IVIG are the most commonly preferred treatments. ${ }^{9}$ Our patient benefitted from IVIG.

Conclusion. Bilateral facial paralysis is a rare condition which requires a detailed investigation. Thus, Sjogren's Syndrome should be taken into consideration in patients with bilateral facial nerve involvement.

Acknowledgment. We would like to sincerely thank Dr. Gokce Kaan Atac, for his review of the radiology images. We would also like to thank Proof-Reading-Service.com [https://www.proof-reading-service. com] for editing and reviewing this manuscript for English language.

\section{References}

1. Goules AV, Tzioufas AG, Moutsopoulos HM. Classification criteria of Sjogren's syndrome. J Autoimmun 2014; 48-49: 42-5.

2. Berkowitz AL, Samuels MA. The neurology of Sjogren's syndrome and the rheumatology of peripheral neuropathy and myelitis. Pract Neurol 2014; 14: 14-22.

3. Colaci M, Cassone G, Manfredi A, Sebastiani M, Giuggioli D, Ferri C. Neurologic Complications Associated with Sjogren's Disease: Case Reports and Modern Pathogenic Dilemma. Case Rep Neurol Med 2014; 2014: 590292

4. Alinezhad N, Habibagahi Z, Ostovan VR. Bilateral facial palsy as the first presentation of primary Sjögren's syndrome: a case report and review of literatüre. Acta Neurol Belg 2020; 120 : 999-1001.

5. Wei Z, Jiaying S, Junhong G. Bilateral facial paralysis as a rare neurological manifestation of primary Sjogren's syndrome: case-based review. Rheumatol Int 2019; 39: 1651-1654.

6. Ashraf V, Bhasi R, Kumar RP, Girija AS. Primary Sjögren's syndrome manifesting as multiple cranial neuropathies: MRI findings. Ann Indian Acad Neurol 2009; 12: 124-126.

7. Hadithi M, Stam F, Donker AJ, Dijkmans BA. Sjogren's syndrome: an unusual cause of Bell's palsy. Ann Rheum Dis 2001; 60: 724-725.

8. Gaudin RA, Jowett N, Banks CA, Knox CJ, Hadlock TA. Bilateral Facial Paralysis: A 13-Year Experience, Plast Reconstr Surg 2016; 138: 879-887.

9. McCoy SS, Baer AN. Neurological Complications of Sjogren's Syndrome: Diagnosis and Management. Curr Treatm Opt Rheumatol 2017; 3: 275-288. 